\title{
Migración en Chile: nuevo desafío para la formación de competencias culturales en estudiantes de enfermería ${ }^{1}$
}

\author{
Soledad Jofré ${ }^{2}$ \\ Claudia Sepúlveda ${ }^{3}$
}

Institución: Universidad de Chile

\section{ENSAYO}

\section{RESUMEN}

En las últimas dos décadas, Chile ha incrementado su tasa de inmigración nacional: la última estimación determinó que, para el año 2014, el 2,3 \% de la población nacional correspondería a migrantes permanentes, lo cual se ha duplicado en los últimos 12 años. Ante este panorama, es necesario que la formación de profesionales de enfermería se adapte con el fin de responder a las nuevas necesidades sociales del país y, consecuentemente, brindar cuidados considerando la diversidad cultural. El siguiente texto invita a reflexionar sobre la preparación brindada a los enfermeros en formación para la entrega de cuidados culturales sensibles, mediante la adquisición de competencias culturales. A partir de las evidencias, incluir el desarrollo de las competencias culturales en los diversos planes de estudio no es tarea fácil, por el contrario, es un desafío inmensurable. En el contexto de la innovación curricular de la carrera de Enfermería de la Universidad de Chile, se están implementando acciones orientadas a formar competencias culturales a través de la inclusión de un curso: de acuerdo con docentes y estudiantes, la experiencia es satisfactoria y necesaria, pero insuficiente para lograr el objetivo esperado. Dado lo anterior, es indispensable que los profesionales de enfermería desarrollen competencias culturales, máxime ante la oportunidad de generar nuevas estrategias que contribuyan al desarrollo de estas y de generar un modelo que permita avanzar en una gestión del cuidado más equitativa, inclusiva y con pertinencia cultural.

Palabras clave: Educación-en-Enfermería, Educación-Superior, Enfermería-Transcultural.

\footnotetext{
${ }^{1}$ Fecha de recepción: 17 de mayo del 2017

Fecha de aceptación: 20 de junio del 2017

${ }^{2}$ Enfermera. Magister en Educación en Ciencias de la Salud, Académico Instructor del Departamento de Enfermería de la Universidad de Chile. Correo electrónico: sjofre@med.uchile.cl

${ }^{3}$ Enfermera. Magister Gestión y Administración en Salud, Académico Instructor del Departamento de Enfermería de la Universidad de Chile. Correo electrónico: csepulvedac@med.uchile.cl
} 


\title{
Revista Electrónica Enfermeria Actual en costa Rica
}

\section{Migration in Chile: a new challenge for the formation of cultural competences in Nursing students ${ }^{1}$}

Institution: University of Chile

\author{
Soledad Jofré ${ }^{2}$ \\ Claudia Sepúlveda ${ }^{3}$
}

\section{ESSAY}

\begin{abstract}
In the last two decades Chile has increased its national immigration rate. The latest estimate determined that by the year $20142.3 \%$ of the national population will correspond to permanent migrants, doubling in the last 12 years. Given this scenario it is necessary that the training of nursing professionals is adapted in order to respond to the new social needs of the country and consequently to be able to provide care considering cultural diversity. The text invites to reflect on the preparation that is given to nurses in training for the delivery of sensitive cultural care, through the acquisition of cultural skills. In the light of the evidence it is no easy task, including the development of cultural competitions in the various curricula it is an immeasurable challenge. In the context of the curricular innovation of the Nursing career of the University of Chile, actions are being carried out aimed at the formation of cultural competitions through the implementation of a course. According to the perception of teachers and students the experience is satisfactory and necessary but still insufficient to achieve the expected objective. It is undoubtedly essential that nurses develop cultural competencies. As teachers we have the opportunity to generate new strategies that contribute to the development of these and to create a model that allows progress in a management of care that is more equitable, inclusive and has cultural relevance.
\end{abstract}

Keywords: Education-Nursing, Education-Higher; Transcultural-Nursing

\footnotetext{
${ }^{1}$ Date of receipt: May 17, 2017

Date of acceptance: June 20, 2017

${ }^{2}$ Nurse. Magister in Education in Health Sciences, Academic Instructor of the Nursing Departament of the University of Chile. E mail: sjofre@med.uchile.cl

${ }^{3}$ Nurse. Magister in Health Management and Administration, Academic Instructor of the Nursing Departament of the University of Chile. E mail: csepulvedac@ med.uchile.cl
} 


\title{
Migração no Chile: novo desafio para a formação de competências culturais nos estudantes de enfermagem ${ }^{1}$
}

\author{
Soledad Jofré ${ }^{2}$ \\ Claudia Sepúlveda ${ }^{3}$
}

Instituição: Universidade do Chile

\author{
ENSAIO
}

\section{RESUMO}

Nas últimas duas décadas, o Chile tem aumentado sua taxa de imigração nacional: a última estimativa determinou que, para o año 2014, 2,3 \% da população nacional corresponderia a imigrantes permanentes, que duplicou nos últimos 12 anos. Ante este panorama, é necessário que a formação de profissionais de enfermagem se adapte com o fim de responder às novas necessidades sociais do país e, consequentemente, brindar cuidados considerando a diversidade cultural. O seguinte texto nos convida a refletir sobre a preparação brindada aos enfermeiros em formação para a entrega de cuidados culturais sensíveis, mediante a aquisição de competências culturais. A partir das evidências, incluir o desenvolvimento das competências culturais nos diversos planos de estudo não é tarefa fácil, pelo contrário, é um desafio incalculável. No contexto da inovação curricular da carreira de Enfermagem da Universidade do Chile, estão sendo implementadas ações orientadas a formar competências culturais através da inclusão de um curso: de acordo com docentes e estudantes, a experiência é satisfatoria e necessária, mas insuficiente para alcançar o objetivo esperado. Devido ao anterior, é indispensável que os profissionais de enfermagem desenvolvam competências culturais, principalmente diante da oportunidade de gerar novas estratégias que contribuam ao desenvolvimento destas e de gerar um modelo que permita avançar em uma gestão de cuidado mais equitativa, inclusiva e com pertinência cultural.

Palavras-chave: Educação em Enfermagem, Educação Superior, Enfermagem Transcultural.

\footnotetext{
${ }^{1}$ Data de recebimento: 17 de maio de 2016 Data de aceitação: 20 de junho de 2017

${ }^{2}$ Enfermeira. Mestrado em Educação em Ciências da Saúde, Instrutor Acadêmica, Departamento de Enfermagem da Universidade do Chile. Correio eletrônico: sjofre@med.uchile.cl

${ }^{3}$ Enfermeira. Magister Gestão e Administração de Saúde, Instrutor Acadêmica, Departamento de Enfermagem da Universidade do Chile. Correio eletrônico: csepulvedac@med.uchile.cl
} 


\section{Revista Electrónica Enfermeria Actual en costa Rica}

\section{www.revenf.ucr.ac.cr}

\section{INTRODUCCIÓN}

En las últimas dos décadas Chile ha incrementado su tasa de inmigración nacional. No obstante, el año 2001 representa un hito, puesto que diversos procesos internacionales posicionaron al país como uno de los principales lugares de destino de la migración en el espacio sudamericano ${ }^{1}$. Sin duda, el desarrollo económico y la posibilidad de un empleo representan el motivo principal para que los migrantes decidan residir en Chile.

La última estimación determinó que para el año 2014, el 2,3 \% de la población nacional correspondería a migrantes permanentes, lo cual no es mayor que la de otros países latinoamericanos, aunque la cifra impresiona ya que duplica la de 12 años atrás (los datos consideraron como base los extranjeros contabilizados en el último censo válido (2002), además de los permisos de permanencia definitiva de los extranjeros que no fueron contabilizados en esa ocasión ${ }^{2}$ ). Desde el 2001, los migrantes que llegan a Chile se caracterizan por contar con una fuerte presencia femenina, indígena y, más recientemente, afrodescendiente. Las principales comunidades residentes la constituyen peruanos $(31,7 \%)$, argentinos $(16,3 \%)$, bolivianos $(8,8 \%)$, colombianos $(6,1 \%)$ y ecuatorianos $(4,7 \%)$. Este nuevo escenario indudablemente plantea un gran desafío tanto para los profesionales de enfermería, como para el país, ya que Chile no cuenta con un proyecto de ley de migraciones con enfoque de derecho, el cual es imprescindible ${ }^{1}$. Al respecto, se debe mencionar que, en los últimos años, desde el área de salud ha existido un avance en la creación de algunos beneficios a los cuales los inmigrantes pueden acceder; sin embargo, es imperioso detenerse en las innumerables variables que están involucradas en los procesos de migración, como la vulnerabilidad migrante por ser un determinante social, las barreras percibidas de acceso a salud, actitudes de racismo, discriminación y xenofobia por parte de la población nativa, para incorporarlas al momento de proporcionar cuidados culturalmente sensibles ${ }^{3,4}$.

Ante este nuevo contexto de diversidad cultural, es imperativo reflexionar sobre el fenómeno que se está viviendo y preguntar: los enfermeros ¿están verdaderamente preparados para brindar cuidados culturalmente sensibles? Y desde las universidades, ¿se está contribuyendo a la formación de enfermeros con competencias culturales?

\section{DESARROLLO}

A través de los años la profesión de enfermería ha ido evolucionando, hasta adaptarse a los diferentes momentos de la historia, por tanto, "es una ciencia y un arte que ha sabido apropiarse del conocimiento para dar respuesta a los diversos retos que se han planteado en el tiempo",

La teoría de la diversidad y la universalidad de los cuidados culturales, desarrollada por Madeleine Leininger en los años 50, intenta responder a ese fenómeno que hoy enfrenta en el país. Como indica McFarland ${ }^{6}$, Leininger en su teoría describe los cuidados culturales como actos de prestación de cuidados de ayuda, apoyo, facilitación o capacitación sintetizados y culturalmente constituidos hacia uno mismo o hacia los demás, centrados en las necesidades evidentes o anticipadas para la salud o el bienestar del cliente o para afrontar discapacidades, la muerte $\mathrm{u}$ otras condiciones humanas. 


\section{Revista Electrónica Enfermeria Actual en costa Rica}

Asimismo, se postula que estos cuidados son diversos y a la vez universales; es decir, sus modelos, valores, simbologías, modos de vida, tienen puntos convergentes y divergentes, además de que deben ser culturalmente sensibles, por lo tanto, es fundamental que los profesionales desarrollen competencias culturales.

En cuanto a las competencias culturales, son definidas por Cabieses ${ }^{7}$ como "el proceso en el cual los profesionales del cuidado de la salud continuamente se esfuerzan por conseguir la habilidad y la disponibilidad para trabajar efectivamente dentro del contexto cultural de la familia, el individuo, o la comunidad". El fenómeno migratorio ya descrito, condiciona al profesional de enfermería al desarrollo de estas competencias para la entrega de cuidados pertinentes y sensibles para el foráneo, máxime considerando la variación y diversidad del origen de los migrantes en los últimos años.

Las competencias culturales, como cualquier otra, requieren desempeños en contextos complejos: como explica Marrero ${ }^{8}$, están compuestas por:

(a) Deseo cultural, que es el interés que demuestra una persona por conocer otras culturas.

(b) Conocimiento cultural, es decir, el aprendizaje que una persona realiza de otras culturas.

(c) Conciencia cultural, mediante la cual, ya conociendo la cultura, es capaz de comprender sus diferencias, haciendo uso de la empatía.

(d) Habilidades culturales, que permite que la persona sea capaz de dar respuesta a las necesidades de quienes pertenecen a otras culturas, según su concepción de la realidad.

(e) Los encuentros culturales, que están dados por la interacción de la persona con distintas culturas.

(f) La sensibilidad cultural, desde la que la persona puede aceptar y respetar al otro en su cultura, lo cual representa el momento en el que se da la comunicación transcultural.

Sin ninguna duda, como académicos una preocupación emergente es formar estas competencias culturales en los estudiantes de pregrado para contribuir, como lo refiere Ibarra ${ }^{9}$, al desarrollo de la aceptación y respeto por las diferencias culturales, la sensibilidad para entender cómo esas diferencias influyen en las relaciones con las personas, y la habilidad para ofrecer estrategias que mejoren los encuentros culturales como requisitos para que el cuidado transcultural en enfermería se pueda consolidar y, finalmente, con ello dar respuestas a las necesidades sociales actuales del país.

Antes de plantear la experiencia local al respecto, es interesante indagar la forma cómo otros países han abordado el fenómeno de la migración desde la formación disciplinar. A nivel internacional, algunos trabajos muestran experiencias relativas a la formación de competencias culturales en distintos contextos: un ejemplo es el estudio realizado en Turquía a 516 enfermeras clínicas con el fin de evaluar la sensibilidad cultural, el cual demostró que la mayoría de participantes experimentaron problemas relacionados con el idioma, el nivel de educación de los pacientes, la percepción de la salud y sobre la enfermedad y las creencias religiosas. Cabe señalar que aquellas enfermeras que habían participado en programas de formación en pregrado y postgrado, demostraron una mayor sensibilidad cultural ${ }^{10}$.

La formación en competencia cultural forma parte de los planes de estudios de enfermería en países como 


\section{Revista Electrónica Enfermeria Actual en costa Rica}

Estados Unidos, Canadá, Reino Unido o Australia, en los que existen algunos hospitales donde, incluso, desarrollan entrenamientos específicos para profesionales ${ }^{11}$. Mientras tanto, en algunos países europeos la realidad es diferente, existe consenso en señalar que en las distintas escuelas de enfermería no hay suficiente formación en cuidado transcultural. Como ejemplo, en España, se plantea dos dificultades fundamentales: una es que no hay tradición en la elaboración y uso de modelos que desarrollen las competencias culturales ${ }^{8}$ y la segunda, según Castrillón ${ }^{12}$, "que el problema de las teorías y modelos disciplinares no son prácticos ni útiles para su uso, pues se consideran discursos amplios y vagos no aplicables a la vida real y, al ser así, no las realizan". A pesar del escenario planteado, existen universidades como las de Oviedo y la Autónoma de Barcelona que ya han puesto en marcha una o dos asignaturas, con la finalidad de incorporar los temas al currículo ${ }^{13}$.

Otro país europeo donde no existe educación en enfermería transcultural ni en los cursos de pregrado o postgrado es Eslovenia. Sin embargo, se ha propuesto a partir del año 2013 un curso electivo en el programa de enfermería de posgrado en la Facultad de Ciencias de la Salud de la Universidad de Primorska con el fin de introducir la enfermería transcultural en el plan de estudios actual ${ }^{14}$.

A nivel latinoamericano, hay poca evidencia sobre el tema. En Brasil, específicamente en la Escola de Enfermagem Aurora Afonso Costa de la Universidad Federal Fluminense, ante el fenómeno migratorio y el incumplimiento de leyes que exigen que los problemas de salud de la población negra, la diversidad cultural y de género se incorporen a los planes de estudios, plantearon como estrategia incluirlos en un curso de pregrado "Diversidad, equidad en salud y cuidados de enfermería" considerando un enfoque de enseñanza-aprendizaje basada en los derechos humanos. La implementación de este curso tuvo un resultado positivo ${ }^{15}$.

Otra interesante experiencia se relaciona con la interacción de un grupo de profesionales de salud con en el pueblo indígena Achuar, en la Amazonía ecuatoriana, en su mayoría, médicos y enfermeros que se desempeñan en acciones de promoción de salud de acuerdo con los preceptos de la medicina occidental. Los profesionales que permanecieron un corto tiempo en el pueblo, expresaron tener apertura al intercambio intercultural, lo que no implica que adquirieran conciencia del sistema de valores y creencias del pueblo Achuar. Además, contaron con información recibida desde las instituciones, la cual tendía a generalizar las características de todas las poblaciones indígenas, dato insuficiente para facilitar el desarrollo de competencias culturales en la entrega de cuidados al pueblo Achuar; sin embargo, quienes llevaban más tiempo en su trabajo en el lugar, parecían tener una mayor sensibilidad cultural en la relación con los indígenas Achuar. Estas experiencias muestran que quienes han avanzado a los encuentros culturales logran más desarrollo de competencias en este ámbito en comparación con quienes se encuentran en un conocimiento o deseo cultural. Los autores, entre sus conclusiones, señalan que es fundamental la formación de competencias culturales en el personal que entrega atención de salud desde los niveles iniciales ${ }^{16}$.

En Colombia en la Universidad Simón Bolívar poseen programas de pregrado en los que incorporan la teoría de la diversidad cultural. No obstante, un estudio que midió la incorporación de las competencias en el perfil de salida, corroboró una gran debilidad en los estudiantes para aplicar adecuadamente en sus acciones las teorías y modelos del entorno y de la cultura del cuidado ${ }^{17}$.

A nivel nacional, la evidencia que dé cuenta del desarrollo de competencias culturales dentro de los planes de estudio de las carreras de Enfermería es escasa. Al respecto, se revisó el perfil de egreso de 43 universidades tanto estatales como privadas: 15 de ellas incorporan términos como "multiculturalidad", "interculturalidad", 


\section{Revista Electrónica Enfermeria Actual en costa Rica}

"perfil sociocultural", "culturas", "arraigo cultural", "diversidad de costumbres"; todas apuntando al desarrollo de competencias culturales. Al revisar la malla curricular, tres universidades incorporan algún curso vinculado directamente con temas de multiculturalidad, de lo cual se colige un incipiente interés por la formación e incorporación de competencias culturales en los planes de estudios ${ }^{18}$.

Como experiencia local se debe señalar que, en el marco de la innovación curricular implementada en la carrera de enfermería de la Universidad de Chile, a partir del año 2014 se comienza a dictar el curso "Educación para la Salud y Multiculturalidad" 19 , el cual se desarrolla durante el cuarto semestre de estudio, de un total de diez. El curso está orientado a que los estudiantes de pregrado logren desarrollar las siguientes subcompetencias:

- Relacionar el concepto de interculturalidad con su rol como profesional de enfermería reconociendo y valorando las diversas necesidades de personas y comunidades.

- Diseñar intervenciones educativas en una comunidad multicultural, incorporando la diversidad como fuente de riqueza individual y colectiva para el aprendizaje grupal.

- Aplicar los conceptos de democracia, inclusión, pluriculturalidad y justicia social en su rol como ciudadano.

- Reconocer en las personas y grupos sociales la capacidad de construir colectivamente respuesta a sus necesidades.

- Argumentar desde el rol profesional su compromiso ciudadano desde una mirada inclusiva, pluralista y democrática.

En la primera versión del curso el 2014, las estrategias metodológicas utilizadas en el desarrollo del curso fueron clases expositivas participativas, talleres y la reflexión respecto de las temáticas abordadas ${ }^{19}$.

Desde la versión 2015- 2016, se incorporó el desarrollo de unidades educativas dirigidas a grupos culturales diversos en los centros de salud familiar en la comuna de Recoleta, promoviendo de esta forma el encuentro cultural ${ }^{20,21}$. Entre las opiniones obtenidas de los estudiantes encuestados al finalizar el curso, se destaca que, a pesar de que el curso es corto, las dinámicas de reflexión son pertinentes, así como la bibliografía, debido a que subrayan la importancia del contenido social del curso. Sin embargo, hay quienes opinan que estas temáticas deberían ser abordadas de manera transversal dentro del currículo. De igual modo, los docentes participantes del curso, a través de un reporte a la comisión de innovación curricular, manifestaron la necesidad de transversalizar el desarrollo de las competencias culturales en el plan de estudios, principalmente en el área del dominio troncal correspondiente a la gestión del cuidado.

Existen también otros cursos que contribuyen al desarrollo de competencias genéricas a través del currículo, como la comunicación efectiva y la relación terapéutica que podrían contribuir a la formación de competencias culturales. Sin embargo, en el desarrollo de estas competencias no se fomenta los encuentros culturales y, si estos ocurren, son accidentales. 


\section{Revista Electrónica Enfermeria Actual en costa Rica}

\section{CONCLUSIONES}

En un país donde los flujos migratorios van creciendo y diversificándose, es indispensable que los profesionales de enfermería adquieran competencias para gestionar cuidados culturalmente sensibles. Al respecto, según Comelles y Bernal ${ }^{22}$, "es indispensable que el profesional de la salud sepa gestionar y analizar significados culturales, comprender la variedad de situaciones en la que se encuentra y ser capaz de relativizar los propios prejuicios".

De igual forma, las instituciones de educación superior pueden contribuir al desarrollo de estas competencias, tal como lo menciona Roca ${ }^{13}$ "el potencial transformador de las instituciones educativas es innegable y la universidad debe convertirse en el motor del cambio social". La enseñanza de enfermeros en formación parece ser el vehículo ideal para que la competencia intercultural se desarrolle dentro de la práctica de futuros enfermeros, con el fin de contribuir de manera importante a una gestión individualizada y pertinente del cuidado que se brinda a personas con diversidad cultural.

Evidentemente el fenómeno de la migración es una oportunidad para generar nuevas estrategias, tal como lo expone Prosen ${ }^{14}$ "es hora de que las escuelas de enfermería utilicen la oportunidad y se conviertan en modelos de excelencia para el sistema de atención de salud al abrazar la diversidad y la competencia cultural en su plan de estudios".

Si bien es cierto, a nivel internacional la evidencias muestra algunas experiencias de incorporación del desarrollo de las competencias culturales a nivel curricular, no son cuantiosas y existe el consenso de que la implementación representa algún grado de dificultad.

Desde la experiencia local, la incorporación del curso "Educación para la salud y multiculturalidad" representa un buen comienzo, dado que incluye la propuesta de la teoría de la diversidad y la universalidad de los cuidados culturales desarrollada por Madeleine Leininger, además de que promueve el encuentro cultural, sin embargo, es insuficiente para el total desarrollo de competencias culturales. En consecuencia, se propone incentivar la formación de la competencia cultural de manera transversal en el currículo, a través de la inclusión y desarrollo de contenidos en todos los cursos del área disciplinar desde el inicio del plan de estudios, asegurando la progresión en la formación de la competencia, desde el estímulo del deseo cultural, hasta los encuentros culturales en los últimos años de la carrera.

Finalmente, es importante reforzar que, en el contexto de migración actual, la formación de competencias culturales parece ya no ser una opción: la enfermería transcultural es esencial en la práctica de los cuidados de enfermería ${ }^{23}$.

Los docentes enfrentan un gran desafío: la oportunidad de generar nuevas estrategias que contribuyan al desarrollo de estas y de generar un modelo que permita avanzar en una gestión del cuidado más equitativa, inclusiva y con pertinencia cultural. 


\section{Revista Electrónica Enfermeria Actual en costa Rica}

\section{REFERENCIAS BIBLIOGRÁFICAS}

1. Rojas N, Silva C. La migración en Chile: breve reporte y caracterización. España: OBIMID; 2016 [Internet]. Disponible en https://www.documentcloud.org/documents/3406604-Informe-Julio-Agosto$\underline{2016 . h t m l}$

2. Departamento de Extranjería y Migración del Ministerio del Interior y Seguridad Pública. Migración en Chile 2005 - 2014. Chile: Ministerio del Interior y Seguridad Pública; [Internet]. 2016. Disponible en http://www.extranjeria.gob.cl/media/2016/02/Anuario-Estad\%C3\%ADstico-Nacional-Migraci\%C3\%B3nen-Chile-2005-2014.pdf

3. Elizalde A, Thayer LE, Córdova, MG. Migraciones sur-sur: paradojas globales y promesas locales. Polis. Revista Latinoamericana. [Internet] 2013; [consultada el 23 de marzo de 2017]; 35. Disponible en http://polis.revues.org/9375

4. Cabieses, B. La Compleja Relación Entre Posición Socioeconómica, Estatus Migratorio y Resultados de Salud. Value in Health Regional Issues [Internet]. 2014[consultada el 23 de marzo de 2017]; 5: 1-6. Disponible en http://dx.doi.org/10.1016/j.vhri.2013.11.001

5. Álvarez D. Enfermería en América Latina: una mirada al horizonte. Avances en Enfermería[Internet]. 2015 [consultada el 23 de marzo de 2017]; 33(2):298. Disponible en http://dx.doi.org/10.15446/av.enferm.v33n2.37032

6. Mc Farland. Teoría de la diversidad y de la universalidad de los cuidados culturales. En: Raile M. editora. Teorías y Modelos de Enfermería. 8a ed. España: Elsevier; 2015. p 410-411.

7. Cabieses B, Bernales M, McIntyre AM. La migración internacional como determinante social de la salud en Chile: evidencia y propuestas para políticas públicas. (2017). Repositorio Universidad del Desarrollo. P 21.

8. Marreno, CM. Competencia Cultural. Enfoque del modelo de Purnell y Campinha-Bacote en la práctica de los profesionales sanitarios. ENE, Revista de Enfermería [Internet]. 2013. [consultada el 23 de marzo de 2017]; 7(2). Disponible en http://ene-enfermeria.org/ojs/index.php/ENE/article/view/278

9. Ibarra TX. Siles J. Competencia Cultural: Una forma humanizada de ofrecer Cuidados de Enfermería. Index de Enfermería[Internet]. 2006 [consultada el 23 de marzo de 2017]; 15(55): 44-48. Disponible en: http://scielo.isciii.es/scielo.php?script=sci_arttext\&pid=S1132-12962006000300010

10. Yilmaz M, Toksoy S, Direk ZD, Bezirgan S, Boylu M. Cultural Sensitivity Among Clinical Nurses: A Descriptive Study. Journal of Nursing Scholarship [Internet]. 2017 49(2):153-161. Disponible en: http://onlinelibrary.wiley.com/doi/10.1111/jnu.12276/abstract;jsessionid=53846EBC546BD3D240FE8E1 708D26F06.f03t03?systemMessage=Wiley+Online+Library+will+be+unavailable+on+Saturday+01 st+Jul 


\section{Revista Electrónica Enfermeria Actual en costa Rica}

$\mathrm{y}+$ from+03.00-09.00+EDT+and+on+Sunday+2nd+July+03.00-

06.00+EDT+for+essential+maintenance.++Apologies+for+the+inconvenience.

11. Plaza del Pino FJ, Soriano E. Formación de los profesionales de enfermería: Cuidar en la sociedad multicultural del siglo XXI. Index de Enfermería [Internet]. 2009 [consultada el 23 de marzo de 2017]; 18(3): 190-194. Disponible en http://scielo.isciii.es/scielo.php?script=sci_arttext\&pid=S113212962009000300011

12. Castrillón Chamadoira, E. La enfermera transcultural y el desarrollo de la competencia cultural. Cultura de los Cuidados [Internet]. 2015 [consultada el 23 de marzo de 2017]; 19, 42; Disponible en http://dx.doi.org/10.14198/cuid.2015.42.11

13. Roca N, Vega C. Diversidad cultural: actitudes, valores y saberes de los estudiantes de enfermería. EMIGRA working papers [Internet]. 2007; (73): 0001-19. Disponible en: https://ddd.uab.cat/pub/emigrawp/emigrawp_a2007n73/emigrawp_a2007n73p1.pdf

14. Prosen, M. Introducing transcultural nursing education: Implementation of transcultural nursing in the postgraduate nursing curriculum. Procedia-Social and Behavioral Sciences [Internet]. 2015; 174: 149155. Disponible en http://www.sciencedirect.com/science/article/pii/S1877042815006916

15. Cruz I. C. Diversidade, Equidade em Saúde e Cuidados de Enfermagem-proposta de disciplina para a graduação em enfermagem na EEAAC/UFF. Boletim NEPAE-NESEN [Internet]. 2015; [Consultado el 25 de marzo de 2017] 11(3). Disponible en http://www.uff.br/jsncare/index.php/bnn/article/view/2780

16. Pulido-Fuentes M, Abad L, Silva MF, Flores JA. Health Competence from a Transcultural Perspective. Knowing how to Approach Transcultural Care. Procedia-Social and Behavioral Sciences[Internet]. 2017 [Consultado el 25 de marzo de 2017] 237: 365-372. Disponible en http://www.sciencedirect.com/science/article/pii/S1877042817300228

17. Lastre G .Introyección en el estudiante de último semestre de las teorías y modelos de formación que fundamentan el programa de enfermería de la Universidad Simón Bolívar. [Tesis maestría] Universidad Nacional de Colombia; 2009 (repositorio). Disponible en http://www.bdigital.unal.edu.co/8584/

18. Universia.cl. (2017). Universia Chile. Portal de las Universidades chilenas. [online] Disponible en: http://www.universia.cl [Consultado el 25 de marzo de 2017].

19. Escuela de Enfermería. Programa de curso Educación para la Salud y Multiculturalidad. Santiago: Universidad de Chile. (2014)

20. Escuela de Enfermería. Programa de curso Educación para la Salud y Multiculturalidad. Santiago: Universidad de Chile. (2015) 


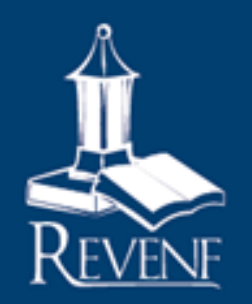

\section{Revista Electrónica Enfermeria Actual en costa Rica}

\section{www.revenf.ucr.ac.cr}

21. Escuela de Enfermería. Programa de curso Educación para la Salud y Multiculturalidad. Santiago: Universidad de Chile. (2016)

22. Comelles JM Bernal M. El hecho migratorio y la vulnerabilidad del sistema sanitario en España. HUMANIAS Humanidades Médicas [Internet]. 2007 [Consultado el 25 de marzo de 2017]; 13: 16-25. Disponible en: http://www.iatros.es/wp-content/uploads/humanitas/materiales/TM13.pdf

23. Vilelas, J. M. D. S., y Janeiro, S. I. D. Transculturalidade: o enfermeiro com competência cultural. Revista Mineira de Enfermagem [Internet]. 2012 [Consultado el 25 de marzo de 2017]; 16(1): 120127.Disponible en http://www.reme.org.br/artigo/detalhes/509 